\title{
System and algorithm for evaluation of human auditory analyzer state
}

\author{
Mykhaylo V. Bachynskiya, Oleksandr Yu. Azarkhov', Dmytro Kh. Shtofele, \\ Svitlana M. Horbatiuk $^{\mathrm{d}}$, Tomasz Ławicki ${ }^{*}$, Aliya Kalizhanova $^{\mathrm{f}}$, Saule Smailova ${ }^{\mathrm{g}}$, Nursanat \\ Askarova $^{\text {h }}$ \\ a Ternopil Ivan Puluj National Technical University, 56 Ruska Str., 46001 Ternopil, Ukraine; \\ ${ }^{\mathrm{b}}$ Pryazovskyi State Technical University, 87500 Mariupol, Ukraine; \\ ${ }^{\mathrm{c}}$ Vinnytsia National Technical University, 95 Khmelnytske Shose Str., 21021 Vinnytsia, Ukraine; \\ d Mykola Pyrohov National Medical University of Vinnytsia, 56 Pyrohova Str., 21018 Vinnytsia, \\ Ukraine; ${ }^{\mathrm{e}}$ Lublin University of Technology, 38A Nadbystrzycka Str., 20-618, Lublin, Poland; \\ ${ }^{\mathrm{f}}$ al-Farabi Kazakh National University, Almaty, Kazakhstan; ${ }^{\mathrm{g}}$ D.Serikbayev East Kazakhstan State \\ Technical University, Ust-Kamenogorsk, Kazakhstan; ${ }^{\mathrm{h}}$ Kazakh National Research Technical \\ University after K. I. Satpaev, Almaty, Kazakhstan
}

\begin{abstract}
The paper discusses questions of human auditory state evaluation with technical means. It considers the disadvantages of existing clinical audiometry methods and systems. It is proposed to use method for evaluating of auditory analyzer state by means of pulsometry to get the medical study more objective and efficient. It provides for use of two optoelectronic sensors located on the carotid artery and ear lobe, Using this method the biotechnical system for evaluation and stimulation of human auditory analyzer stare wad developed. Its hardware and software were substantiated. Different modes of simulation in the designed system were tested and the influence of the procedure on a patient was studied.
\end{abstract}

Keywords: auditory analyzer, functional state, biotechnical system, algorithm, pulse characteristic, simulation modes

\section{INTRODUCTION}

Auditory analyzer is an important part of human sensory system, it's functional state determines the ability of a person to perform different specific kinds of activity. It has been studied as a receiver and a source for sound and electromagnetic waves ${ }^{1,2,3,4}$. We suggest using pulse diagnostics in order to take into account the physiological background of auditory system functioning.

One of the functions of pulse diagnostics is to obtain information about the status of blood supply and blood circulation in various organs and tissues of the human body by non-invasive means. As the circulatory system covers the entire body, it gives the physician an opportunity to form a picture of the qualitative and quantitative expression of status of any organ or system of the human body. Therefore, in this case we do not require detailed model for signal processing in the auditory analyzer such as ${ }^{5}$.

This allows to take the method for obtaining of acoustic analyzer diagnostic indicators by analyzing pulse waves recorded from the ear lobe and carotid artery ${ }^{6}$ as a foundation of a system for state evaluating of the auditory analyzer. Existing methods of otorhinolaryngology study of human hearing are subjective and have significant drawbacks. First of all, the considerable errors are caused by subjective character of evaluation, especially while determining the moment when person begins to hear a sound, which usually is formed by the doctor. This leads to a significant reduction of informative and diagnostic capabilities of assessment of the factual functional state of the auditory analyzer.

\footnotetext{
*t.lawicki@pollub.pl, phone 48815384311
} 
Modern clinical and household audiometers, despite of their wide dissemination, are also not devoid of the disadvantage mentioned. Methods of audiological research suggest that the patient must respond to the presence or absence of auditory perception of sound self-consciously and independently ${ }^{7,8,9,10}$. This contributes the process of measuring some elements of subjective perception, the possibility of fraud, causes a significant error in researches related to inaccurate determination of the hearing level.

\section{METHODS}

In humans in different physiological and functional states the characteristics of pulse wave, as a result of the operation of various control systems, undergo significant qualitative and quantitative changes ${ }^{11}$.

One of the most accessible and popular methods for registering a pulse in the peripheral cardiovascular system is photoplethysmography (PPG) based on the measurement of the transparency of the subcutaneous space. The essence of the method is the analysis of light passed through body tissue. For such lighting a source of infrared light is usually used.

The spectrum of light absorption in the blood is in the infrared radiation range, i.e. it has low transparency for IR radiation; and each heart beat changes the pressure in the blood vessels, which changes transparency in turn. A transparency is determined primarily by the volume and composition of the blood. Thus, the greater portion of the blood supply leads to decrease of its transparency in infrared spectrum ${ }^{12}$.

Using the PPG method for heart rate recording, a part of the standard heart rate variability analysis enables to estimate the shape of the pulse wave state of the cardiovascular system and organism regulatory systems. Mathematical processing of high-quality PPG signal allows to study other cyclic processes in the body, and the response to external factors $^{13}$.

Modern biomedical equipment allows to obtain high quality PPG signal. Requirements for technical support include the ability to register a constant component of the signal, and low sensitivity of system to external interference. Thus, medical and technical requirements may be characterized by the following parameters ${ }^{13}$ : frequency spectrum of the recorded signal $-0 . .25 \mathrm{~Hz}$; irregularity of frequency characteristic $-2 \mathrm{~dB}$; inhibition of ambient light and electric circuit interferences $-60 \mathrm{~dB}$; number of gradations of digital signal -28 bits; digitizing frequency $-1,1 \mathrm{kHz}$.

To obtain such characteristics the portable device was developed, which registers the pulse characteristic from the ear lobe and carotid artery. It has a digital amplifier with feedback and digital filtering. It can make PPG pulse wave shape clearly visible.

When choosing a method for determining the signal spectrum it is necessary to give preference to methods that minimize the effects associated with the discrete sampling.

To analyze the frequency and shape of the pulse wave it is advisable to apply method for nonlinear systems, which is regardless of the signal model, which is destined to study the structure of the time series, and combines the advantages of many other methods, such as Fourier analysis and regression analysis.

\section{SYSTEM AND ALGORITHM DESIGN}

To get rid of subjectivity in evaluating the state of the auditory analyzer we must abandon conscious participation of the patient in the study. This can be achieved by measuring and evaluating objective physiological parameters related to the sound perception.

It is known that human auditory analyzer functioning is associated with the elasticity of blood vessels that nourish $\mathrm{it}^{14}$. It leads to a change of a pulse characteristics shape and the character of its spectral components. One of the most informative parameters of the ear sensitivity is a bandwidth of pulse signal registered at the operator earlobe.

At the same time, according to ${ }^{6}$, the pulse characteristics of the carotid artery that feeds blood vessels of auditory system, is characterized with a tolerant value for upper range frequency $F_{U C A}$ to its lower frequency $F_{L C A}$ ratio, and does not depend on the state of the auditory system.

Thus, the method of objective investigation of hearing based on the analysis of changes in the sensitivity of the ear to the level and frequency of the external audio signal, includes acoustic signal of fixed frequency and variable level providing into the external ear canal. The method is that the pulse characteristic of carotid artery is previously recorded, the extreme values of frequency of its pulse spectrum are determined and recorded, and then the coefficient $A_{C A}{ }^{6}$ is calculated:

$$
A_{C A}=\frac{F_{U C A}}{F_{L C A}} .
$$


A bandwidth of pulse signal registered at the operator earlobe is limited with values $F_{E I}$ and $F_{E 2}$. Similarly to (1), we can find the expression for the coefficient of the extreme values of frequency of pulse characteristic spectrum, registered at the ear lobe $A_{E L}$ :

$$
A_{E L}=\frac{F_{U E L}}{F_{L E L}},
$$

where $F_{U E L}$ and $F_{L E L}$ are appropriately upper and lower frequency range borders of pulse characteristic taken from the ear lobe.

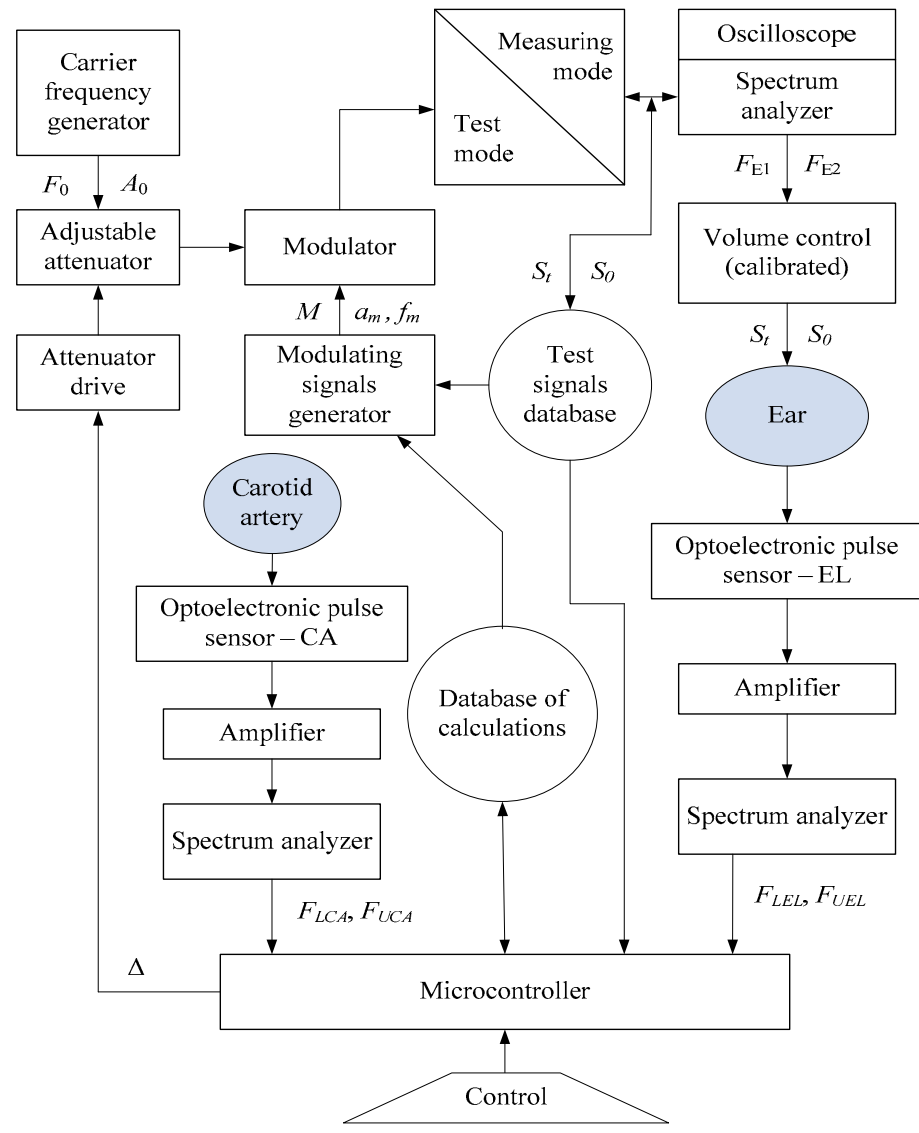

Figure 1. Scheme of the system for evaluation of auditory analyzer state.

Registering the output (zero) frequency we can find the level when the condition of equality of signal spectra from carotid artery and ear lobe is satisfied $\left(A_{C A}=A_{E L}\right)$, based on the calculated value of $A_{E L}$. As an option to determine the pathology of hearing, the deflection of values $A_{E L}$ and $A_{C A}$ for the different frequencies of signal can be used.

The implementation of the described method is achieved by means of a system for the assessment of auditory analyzer state (Figure 1) and the algorithm of its performance (Figure 2). The scheme has the following denotation:

$F_{0}, A_{0}$ - respectively the frequency and amplitude of the carrier frequency;

$f_{m}, a_{m}$ - respectively the frequency and amplitude of the modulating frequency;

$M$ - type of modulation;

$S_{t}$ - test signal;

$S_{0}$ - investigation (work) signal that goes directly into the ear;

$F_{\mathrm{E} 1}, F_{\mathrm{E} 2}$ - respectively lower and upper frequencies of the pulse characteristics range of ear vessels;

$F_{L C A}, F_{U C A}$ - respectively lower and upper frequencies of the range of signal taken from the carotid artery;

$F_{L E L}, F_{U E L}$ - respectively lower and upper frequencies of the range of the pulse signal taken from the ear lobe. 


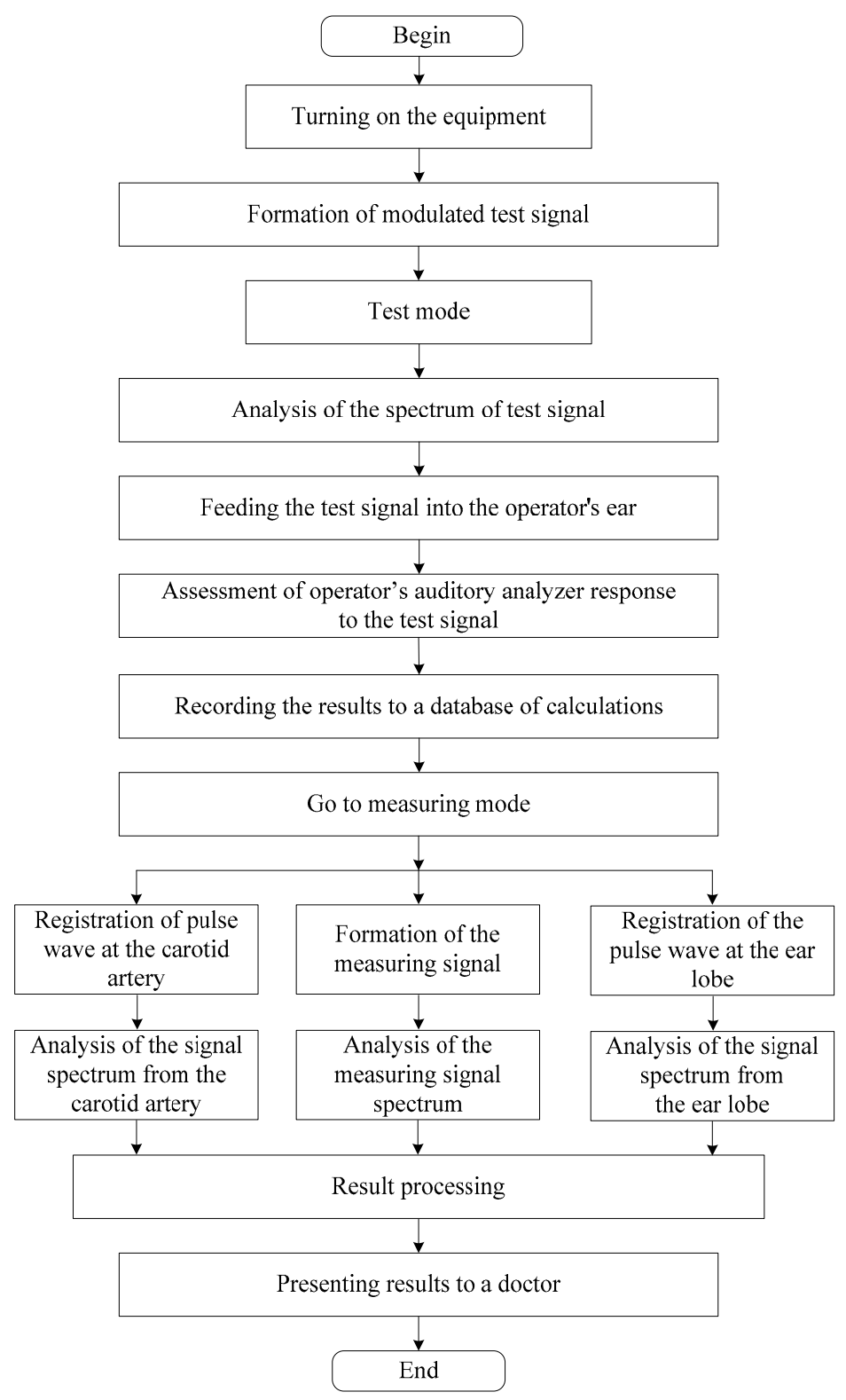

Figure 2. Generalized algorithm for system for evaluation of the auditory analyzer state.

Table 1. Information on video and audio files that can accompany a manuscript submission.

\begin{tabular}{|l|l|l|}
\hline \multicolumn{1}{|c|}{ Item } & \multicolumn{1}{|c|}{ Video } & \multicolumn{1}{c|}{ Audio } \\
\hline File name & Video1, video2, .. & Audio1, audio2, $\ldots$ \\
\hline Number of files & $0-10$ & $0-10$ \\
\hline Size of each file & max. $5 \mathrm{mb}$ & max. 5mb \\
\hline File types accepted & $\begin{array}{l}\text {.mpeg, .mov (Quicktime), .wmv } \\
\text { (Windows Media Player) }\end{array}$ & .wav, .mp3 \\
\hline
\end{tabular}




\section{RESULTS}

During the study of the discussing system there was found that the carrier frequencies of $131 \mathrm{~Hz}, 147 \mathrm{~Hz}, 165-$ $169 \mathrm{~Hz}$ can cause depression (at any frequency of binaural beats), but "pink" (flicker) noise and carrier frequencies above $330 \mathrm{~Hz}$ help to negate this effect and overcome its consequences ${ }^{15}$.

It was shown that the impact of electromagnetic fields on humans is very specific. There exist the separated frequencies, causing abrupt changes in the functioning of organism. Such frequencies are called bioeffective ${ }^{16}$. The response to them may be different: both positive (in terms of transition of body condition closer to the optimum), or negative. The sharp frequency response of the human body were observed at frequencies: $0.02,0.05-0.06,0.1-0.3,0.5-$ $0.6,5-6,8-12 \mathrm{~Hz}$.

For stimulation, various forms of audio signals are used: sinusoidal (pleasant for perception), rectangular (perhaps the most effective, although it is unpleasant to the ear), triangle (it is closer to the natural forms of audio signals), sawtoothed (has activating effect), as well as various forms of arbitrary signals, including "pink" noise (which is similar to the sound of the sea, waterfalls, rain, deciduous forest), "white" noise (like a TV antenna noise when turned off) (Figure 3). It is also known that Brownian motion of the hair bundle causes to improve the sensitivity of mechanoelectrical transduction in auditory analyzer ${ }^{17}$.

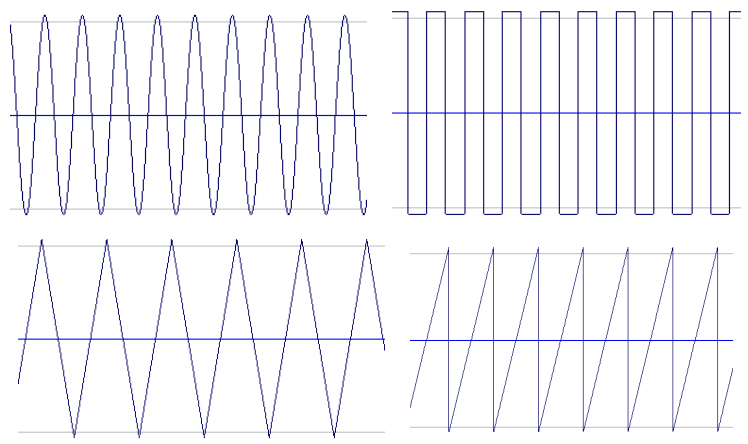

Figure 3. Different shapes of audio waves (based on ${ }^{12}$ ).

Rectangular signal is effective to divert the mind from extraneous thoughts and help to achieve quickly altered states of consciousness ${ }^{18}$. The impact of "pink" noise helps to overcome depression, to distract out from negative thoughts, to achieve a state of relaxation.

It is important to make a subjective selection, all forms are to be tried.

Volume is adjusted individually by means of a regulator.

To achieve higher efficiency, various modes of stimuli application and their combinations are used ${ }^{15}$ :

1. Synchronous mode - simultaneous feeding incentives from right and left side. It ensures synchronization of the functional activity of the right and left hemispheres. This mode does not occur in everyday life ${ }^{19}$.

2. Asynchronous mode - alternate feeding signals from right and left.

3. Dissociating mode - alternate feeding of auditory and visual stimuli. It is intended to isolate the perception of sensory information that leads to the attention exacerbation, increases the ability to highlight the main things, problem-solving ability ${ }^{20}$. This mode is used for reducing the strength of the nervous processes.

4. Advanced mode - alternate turning on sensory stimuli with the change of lateralization of feeding. It increases the resolution of analyzers activating different zones of cortex ${ }^{21}$.

5. Alternating mode - stimulation with different frequencies from right and left. It is indispensable in stressful situations, when the hemispheric asymmetry coefficient changes dramatically. Using this mode, it is possible to have a different impact on the right and left hemisphere activity, while the profile of functional hemispheric asymmetry is pre-defined.

6. Mode with the acoustical image offset - in this mode the subjective sensation of sound perception is displaced from the midline (usually located in the crown, sometimes at the nose and chin) in the direction of that ear where sound stimuli are fed ahead. Person has the feeling of sound rotation in his head, which contributes to more rapid enter the altered state of consciousness (Figure 4). 


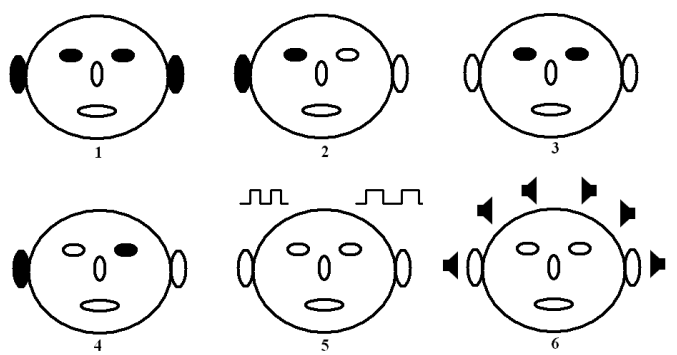

Figure 4. Stimulation modes (based on ${ }^{12}$ ).

To stimulate the orienting reflex (novelty reflex) in order to "capture the brain and distract it from the surrounding reality" a rapid change of the stimulation frequency is used. It is also important in process of central auditory system maturation ${ }^{22}$.

The short procedure duration (7-15 minutes) is used for the purpose of activating the excitation systems of diencephalon and mesencephalon and to increase the motor activity ${ }^{15}$.

The long duration (25-30 minutes) leads to a reduction of physical activity, to the progress of diffuse inhibition processes.

The further increase of the procedure duration (35 minutes and more) leads to the progress of phase activity inhibition processes and to rise of the plastic tone.

The manifestation of the described effects depends on the initial psycho-physiological state of man.

We can argue that the short sessions (15-20 minutes) two or three times a day are more effective than one long session. In any case, the rhythmic impact on the brain lasting more than 45 minutes is not recommended, because in general the brain ceases to follow the light and sound stimuli, and sometimes exceeding 45-minutes threshold may lead to diffuse inhibition of the cerebral cortex (the sleepy twilight state) that passes only after natural sleep. It is advisable to carry out the stimulation daily. At least, the breaks should not exceed three days.

Thus, using both spectral analysis of pulse waves recorded at various points of the operator's body and direct method of formation of testing and measuring signals for evaluation of auditory analyzer the biotechnical system was developed, which provides an enhanced level of diagnostic informational content, efficiency of measurements ${ }^{25,26,27}$, periodic or continuous monitoring of the human auditory analyzer.

\section{CONCLUSIONS}

1. Clinical methods for evaluating of human auditory analyzer state are subjective and sometimes lead to false results of medical study.

2. To avoid the influence of subjective perception the method for evaluating of auditory analyzer state by means of pulsometry was used.

3. Based on this method, the biotechnical system for evaluation of auditory analyzer state was designed. It includes carrier frequency generator, adjustable attenuator, modulator, spectrum analyzers, amplifiers, optoelectronic pulse sensors, appropriate databases, and control block.

4. The principles of system functioning were discussed and the generalized algorithm for the system was developed.

5. Different modes of simulation in the designed system were tested. To achieve higher efficiency of the system, various modes of stimuli and their combinations are to be used. Six basic modes were selected and studied. The duration of the procedure was substantiated.

6. The developed system provides an enhanced level of diagnostic informational content, efficiency of measurements, periodic and continuous objective monitoring of the human auditory analyzer. 


\section{REFERENCES}

[1] Frey, A. H., "Human auditory system response to modulated electromagnetic energy," Journal of Applied Physiology 17(4), 689-692 (1962).

[2] Kemp, D. T., "Stimulated acoustic emissions from within the human auditory system," The Journal of the Acoustical Society of America 64(5), 1386-1391 (1978).

[3] Lonsbury M., Brenda L., et al., "New approaches to the evaluation of the auditory system and a current analysis of otoacoustic emissions," Otolaryngology - Head and Neck Surgery 112(1), 50-63 (1995).

[4] Özgür, A., et al., "Effects of chronic exposure to electromagnetic waves on the auditory system," Acta Otolaryngologica 135(8), 765-770 (2015).

[5] Dau, T., Püschel, D. and Kohlrausch, A., "A quantitative model of the "effective" signal processing in the auditory system. I. Model structure," The Journal of the Acoustical Society of America 99(6), 3615-3622 (1996).

[6] Klimashov, B. M., "Objective estimation of the human auditory system state from pulsation," Biomedical Engineering 45(1), 19-21 (2011).

[7] Wooles, N., et al., "Comparison of distortion product otoacoustic emissions and pure tone audiometry in occupational screening for auditory deficit due to noise exposure," The Journal of Laryngology \& Otology 129(12), 1174-1181 (2015).

[8] Rourke, R., Bromwich, M. and Chan, D., "iPad audiometry in Canada's North: A portable and cost-effective method for hearing screening," Otolaryngology - Head and Neck Surgery 151(1), 104-104 (2014).

[9] Brennan, J., Christopher, G. and Eikelboom, R. H., "Diagnosis of hearing loss using automated audiometry in an asynchronous telehealth model: A pilot accuracy study," Journal of Telemedicine and Telecare 1357633X16641552, (2016).

[10] Kocchar, A., et al., "Role of audiological screening (pure tone audiometry and distortion product otoacoustic emissions) in patients undergoing concurrent chemoradiation for head and neck cancers," SRMS Journal of Medical Sciences 1(1), 27-32 (2016).

[11] O'Rourke, M. F. and Gallagher, D. E., "Pulse wave analysis," Journal of Hypertension Supplement 14, 147-158 (1996).

[12] Yamashina, A., et al. "Validity, reproducibility, and clinical significance of noninvasive brachial-ankle pulse wave velocity measurement," Hypertension research 25(3), 359-364 (2002).

[13] Berezhnoy, V. N. "Pulse wave." NeuroLab.ru (2010): http://www.neurolab.ru/ru/menu/papers/pulse_wave

[14] Dallos, P., [The auditory periphery biophysics and physiology] Elsevier, (2012).

[15] Golub, Ya. V. and Zhyrov, V. M., [Medical and psychological aspects of application of light and sound stimulation and bio-feedback] KERI, S.-Petersburg, (2007).

[16] Khabarova O. V., "Bioeffective frequencies and their rela-tion to own frequencies of living organisms," Biomedical Technologies and Radioelectronics 6, 56-66 (2002).

[17] Jaramillo, F. and Wiesenfeld, K., "Mechanoelectrical transduction assisted by Brownian motion: a role for noise in the auditory system," Nature Neuroscience 1(5), 384-388 (1998).

[18] Mann, J., [Awakening Mind I (Creating sound and light sessions on advanced programmable mind machines)], Enlightened Enterprises, (2005).

[19] Gazzola, V., Aziz-Zadeh, L. and Keysers, C., "Empathy and the somatotopic auditory mirror system in humans," Current Biology 16(18), 1824-1829 (2006).

[20] Picton, T. W., Hillyard, S. A. and Galambos, R., Habituation and attention in the auditory system," Auditory System. Springer Berlin Heidelberg, 343-389 (1976).

[21] Claude, A., et al. "“What" and "where" in the human auditory system," Proceedings of the National Academy of Sciences 98(21), 12301-12306 (2001).

[22] Ponton, C. W., et al., "Maturation of human central auditory system activity: evidence from multi-channel evoked potentials," Clinical Neurophysiology 111(2), 220-236 (2000).

[23] Valtchev, V. S., Teixeira, J. P. and Pavlov, S., „Energy harvesting: an interesting topic for education programs in engineering specialities," Internet, Education, Science (IES-2016), 149-156 (2016).

[24] Kostishyn, S., Tymchyk, S., Vyrozyb, R., Zlepko, A. and Pavlov, V., "Design features of automated diagnostic systems for family medicine," Proc. of 13th International Conference on Modern Problems of Radio Engineering. Telecommunications and Computer Science (TCSET), (2016). 
[25] Sawicki, D., Kotyra, A., Akhmetova, A., Imanbek, B. and Suleymenov, A., "Wykorzystanie metod optycznych do klasyfikacji stanu procesu współspalania pyłu węglowego i biomasy,", Rocznik Ochrona Środowiska 18, 404-415 (2016).

[26] Timchenko, L., Yarovyi, A., Kokriatskaya, N., Nakonechna, S., Abramenko, L., Ławicki, T., Popiel, P., Yesmakhanova, L., "The method of parallel-hierarchical transformation for rapid recognition of dynamic images using GPGPU technology," Proc. of SPIE 10031, 1003155 (2016).

[27] Wójcik, W; Golec, T; Kotyra, A; et al., "Concept of application of signals from fiber-optic system for flame monitoring to control separate pulverized coal burner," Photonics Applications in Astronomy, Communications, Industry, and High-Energy Physics Experiments Ii 5484, 427-431 (2004). 